\title{
Electrophysiological Testing of Eyes with Opaque Media
}

\author{
N. R. GALLOWAY \\ Nottingham
}

\begin{abstract}
Summary
Electrodiagnostic tests as applied to the eye are now widely used in the investigation of patients with inherited retinal degenerations but their value in patients with opaque media is perhaps less well recognised. The choice of stimulus is all important and a flash VEP and flash ERG probably provide the best combination of tests at present. A comparison with the fellow eye, if it is normal, greatly improves the value of the result. A pitfall of this type of test is its failure to detect amblyopia and a carefully recorded history is important in helping to interpret the traces.
\end{abstract}

Now that surgical techniques for the removal of opaque media from the eye have been developed to a high degree, it is often necessary to make an asssessment of the function of the retina and visual pathway when it is not possible to obtain a clear view of the fundus either by direct or indirect ophthalmoscopy. A number of techniques are available for this purpose. An afferent pupillary defect is a useful indication of severe retinal damage. This is probably the simplest and most important of all tests of retinal function in the presence of opaque media. If the other eye is normal then the consensual reaction of the good eye should be noted if there is iris damage on the affected side. The pupil reaction is less helpful in the elderly and in diabetic patients. In these cases the pupil may be small or react relatively poorly even in the presence of good retinal function. On the other hand a normal brisk pupil reaction may be preserved even when there is quite extensive retinal damage but this only applies if the macula region is spared.

\section{Psychophysical Tests of Retinal Function through Opaque Media}

The light projection test is a good measure of peripheral retinal function or damage to the visual pathway although it may be upset by very dense opacities especially vitreous haemorrhage. The use of the Maddox rod is often neglected in this respect. The patient is asked if the rod appears broken up or whether it has a gap in it. If the patient is a good observer then some assessment of foveal function can be made by this means. Alternatively the beam of the slit lamp can be used in the same way by forming it into a narrow slit during attempted examination of the fovea with the triple mirror or +90D lens. Another test of retinal function which can be used in the presence of opaque media is known as Blue Field Entoptoscopy. This test relies on the phenomenon of seeing ones own leucocytes flowing in the perifoveal capillaries. The retina must be diffusely illuminated, a situation provided already by many dense cataracts, and with sufficient illumination a number of 'flying corpuscles'

Correspondence to: N. R. Galloway F.R.C.S., Eye Department, Queens Medical Centre, University Hospital, Nottingham

Presented at the Annual Congress of the Ophthalmological Society of the United Kingdom, April, 1988. 
can be seen moving around in the centre of the visual field. The phenomenon is best observed with blue light. The clinical value of the test was originally appreciated by Scheerer. ${ }^{1}$

A study in pre- and post-operative cataract patients has demonstrated that it is a good predictor of post-operative visual acuity. ${ }^{2}$ The Purkinje test is another test of retinal function which relies on an entoptic phenomenon. If a bright light source is shone onto the sclera and moved about, a rather dramatic view of the shadow of the retinal blood vessels can be seen by the patient. This view of ones own retinal vasculature is impaired if the function of the underlying retina is defective and it can be seen in the presence of opaque media. Yet another test is known as Two Point Discrimination. This assesses the patients ability to discriminate two point sources of light in different parts of the visual field. Both Two Point Discrimination and the Purkinje tests do not seem to fare as well as Blue Field Entoptoscopy when their performance is compared in patients with opaque media. ${ }^{2}$ These tests.which rely on the detection of entoptic phenomena are necessarily highly subjective and reliant on the patient's observations. The application of laser interferometry is an attempt to overcome this difficulty. Laser interference fringes can be projected onto the retina when the media are opaque, especially in the presence of irregular corneal opacities, and may be discerned quite clearly by the patient. A visual acuity measurement can be made by changing the spacing of the fringes. Even this test provides subjective difficulties because the fringes are seen amongst a jumble of other patterns and are not seen at all when the opacities are dense. ${ }^{3,4}$

Since many of these tests have the disadvantage of being subjective and reliant on the patient's ability to describe what they see, there is a need to use more objective methods if accurate measurements of retinal function are to be made. Two truly objective tests are provided by ultrasonography and electrodiagnostics. Ultrasound measures morphological changes and electrodiagnostics measure functional changes, and thus the two tests compliment one another. Here we shall be concerned with the way in which electrodiagnostic techniques can be used to assess retinal function through opaque media.

\section{Electrodiagnostic tests}

Much of the earlier interest in electrodiagnostics as applied to ophthalmology concerned the origin of the different components of the responses and subsequently the manner in which these various responses were affected by disease. The manner in which the responses are affected by opaque media has had less attention in spite of its potential importance. It is possible to record a normal Electroretinogram (ERG) or Visually Evoked Potential (VEP) through a very dense opacity in the absence of other pathology as long as the right sort of stimulus is used. Figure 1 shows the ERGs from each eye of a patient who had suffered a contusion injury to his left eye in childhood and subsequently developed a mature cataract on that side. The other eye was normal. The fact that a normal response is obtained through the mature cataract indicates that the retina

$$
\text { D.B. } 26.1 .82
$$

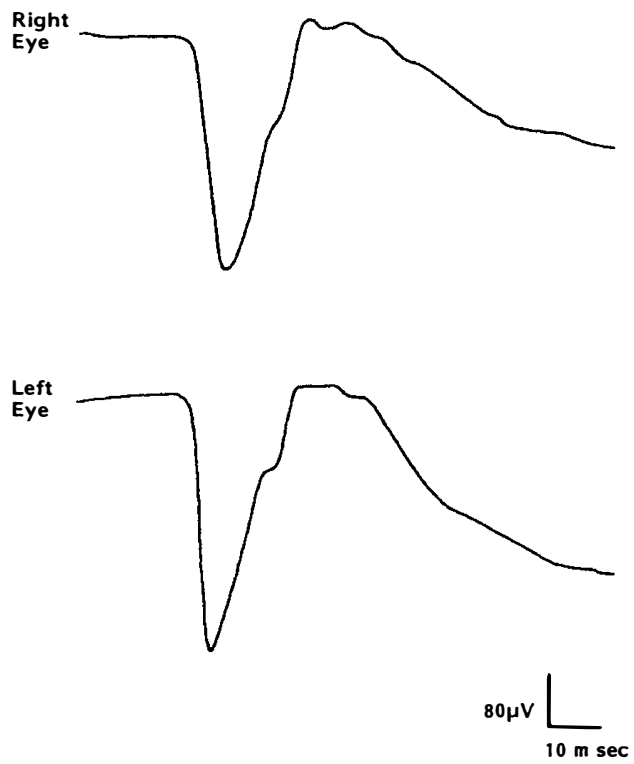

Fig. 1. Normal ERGs from a patient with a mature cataract in the left eye only. 
is not degenerate or detached and shows how little the ERG is affected by opaque media. Electrodiagnostic studies have the possibility of providing both a qualitative and a quantitative measure of retinal activity. A number of different techniques have been developed for measuring the various electrical changes produced in the visual pathway, but only certain types of electrical test are applicable when the optical media are opaque.

\section{Evoked responses}

Electrical responses are evoked from the retina and visual pathway by stimulating the retina with light. Other stimuli can be used but they have not yet been exploited in clinical ophthalmology. The light stimulus can be in the form of a single flash or multiple flashes at different frequencies. The flash may be diffuse (unstructured) or it may be a pattern (structured). Patterned stimuli are scattered by opaque media and are therefore of limited value in this situation. The flash ERG is normally recorded in our own clinic by stimulating, with a wide field, diffuse flash using an electrode which hooks over the lower lid. This type of electrode, if carefully placed, is unlikely to damage the cornea. Contact lens electrodes should be avoided, if possible, in recently injured eyes. The risk of damaging the eye has to be balanced with the more accurate and noise free recording that contact lenses can give. Responses from the occipital cortex are recorded from the back of the scalp (the VEP). Certain important principles for interpreting these responses must be mentioned at this point.

\section{Origins and significance of evoked responses}

It is important to bear in mind that severe retinal damage alone may prevent normal electrical changes from occurring over the occipital cortex. This is to say that abnormalities in the latency and amplitude of the VEP do not specifically signify optic nerve disease as is sometimes thought. Extensive macula degeneration may impair the amplitude of the VEP recorded from the occipital cortex just as much as optic nerve compression or inflammation. It is also important to remember that, unless special techniques are employed, the flash ERG does not reflect localised focal damage and it may remain normal in the presence of macula degeneration. ${ }^{5}$

Furthermore, a normal flash ERG can be obtained even if the optic nerve is severed because the flash ERG, unlike the response to a pattern stimulus, arises distal to the ganglion cells; as long as the receptors, bipolars and surrounding elements are intact, a normal ERG can be produced. ${ }^{6}$ An abnormal ERG may be produced by retinal ischaemia from any cause or extensive degenerative changes. A completely abolished ERG is seen when the retina is totally detached or in patients with retinitis pigmentosa even at a relatively early stage. ${ }^{7}$ The VEP is, of course, abolished by severing the optic nerve. When the retina and visual pathway are intact the flash VEP reflects predominantly macular function probably because of the distribution of connections from the macular region to the occipital cortex. ${ }^{8}$ This effect appears to have some clinical value when used in the presence of opaque media (see below). The relatively generous representation of the macula at the posterior part of the occipital cortex is close to the site of the midline electrode on the surface of the scalp. The ERG can be used to assess cone function by producing a response from a single standard bright flash under light adapted conditions. ${ }^{9}$ The measurement of the response to a standard bright flash flickering at 30 flashes per second against a constantly illuminated background of $5-10 \mathrm{ft}$ $L$ is also effective in isolating the cone response. ${ }^{10}$ The exact definition of this 'standard bright flash' is at present under discussion by an international panel which is considering the important question of standardisation of the ERG. Although the flicker ERG might be expected to provide a good test of macular function through opaque media this particular application does not seem to have been exploited clinically. Detail of how disease of the visual pathway can cause specific changes in the ERG and VEP will not be considered in this review but we need to know how opaque media themselves can modify the recorded electrical response. 
How do opaque media alone modify the response?

The normal ERG shows characteristic modification when neutral density filters are used to vary the strength of the light stimulus. The ' $a$ ' wave and ' $b$ ' wave both increase in size as the light strength increases but the ' $b$ ' wave becomes saturated above a certain level of illumination and the 'a' wave continues to increase. The latency of the ' $a$ ' wave and the ' $b$ ' wave diminishes as the light intensity increases. For clinical purposes it can be taken that a maximal ' $b$ ' wave is reached when the latency of the first negative peak of the ERG is as short as $12 \mathrm{msec}$. Knighton and Blankenship have emphasised the value of recording an intensity response series in each case, when examining patients with opaque media. ${ }^{11}$ The light attenuating effect of opacities in the media can vary between the equivalent of 0.0 to $3.0 \mathrm{log}$ units of neutral density filter, but a neutral density filter cannot mimic exactly the effect of opaque media. For example, although opaque media including cataract may reduce the size of the ERG, it has long been recognised that some patients with cataracts have a larger than normal ERG (The "Ganzfeld effect"). It has been suggested that this may be due to a light scattering effect of the opacity. ${ }^{12,13}$ Figure 2 shows the ERG from a patient with a mature cataract in the left eye. The amplitude of the response is considerably larger than that from the normal right eye in this particular case.

The varied effect of opaque media on vision has been investigated by a number of means. Attempts have been made to mimic the opacities by artificial means and thereby produce an experimental model using normal subjects. ${ }^{14,15,16}$. This type of study has not so far extended into the field of clinical electrodiagnosis.

The flash VEP may be reduced in the presence of a dense cataract but the reduction is slight. Copenhaver and Perry showed that the flash VEP was more affected by neuroretinal lesions than by medial opacities. They also showed that high refractive error (above 10 dioptres) may impair the flash VEP. ${ }^{17}$ It seems reasonable that if the stimulus is bright enough to give a maximal ' $b$ ' wave on the ERG through opaque media, then it should produce a normal VEP, in the presence of a normal visual pathway. Such a stimulus needs to give an illumination of at least $50 \mathrm{~cd} / \mathrm{m}^{2}$. When recording the ERG through opaque media a 'bright flash' technique has certain merits; apart from ensuring a maximal response it can obviate the need for previous dark adaptation or even for dilating the pupils. This is because the response is already maximal and not greatly altered by mydriasis or adaptation. The same seems to apply for the VEP at least as regards pupil size. ${ }^{18,19}$ The problem of pupil diameter might be of special importance in patients with opaque media because quite often the pupil in these cases is bound down by synecheae or otherwise distorted by trauma and inflammation.

When recording the VEP, the average of many responses is examined and repeated stimuli are therefore required. If these are too bright then light adaptation occurs and

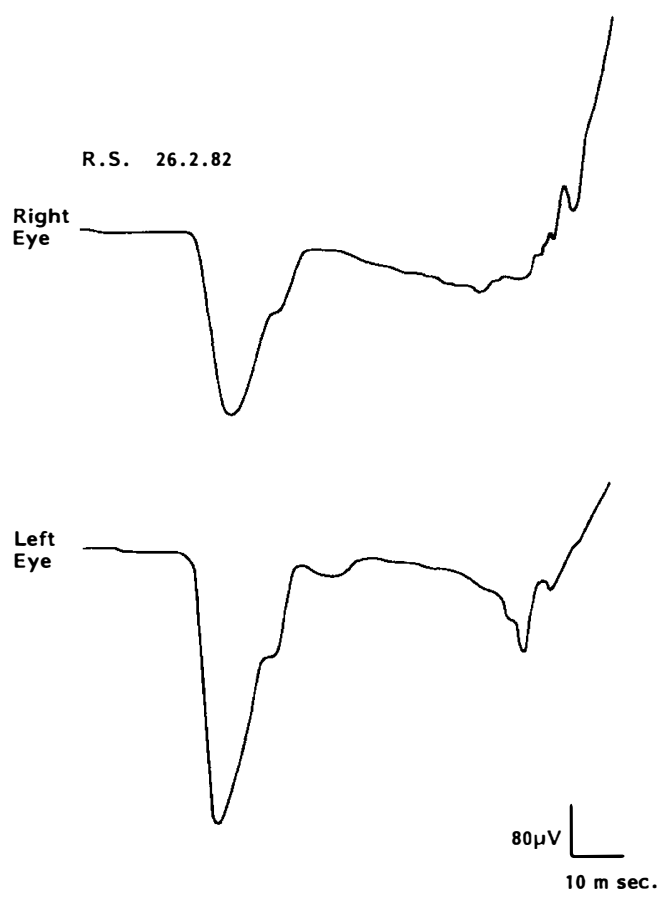

Fig. 2. ERG recorded from a patient with unilateral cataract showing a larger response from the affected side. 
the response becomes smaller and thus an error is introduced into the averaged result. These factors need to be taken into account especially if the ERG and VEP are being recorded simultaneously using the same stimulus. Thompson and Harding used a xenon flash stimulus of stepped intensities from 68 to $483 \mathrm{~cd} / \mathrm{m} 2 / \mathrm{sec}$ to assess visual function in 21 patients with unilateral cataracts. They concluded that the effect of the cataract alone on the amplitude and latency of the flash VEP was minimal and corrected by increasing the brightness of the stimulus from 68 to $96 \mathrm{~cd} / \mathrm{m} 2 / \mathrm{sec}^{20}$

One approach to recording through opaque media has been to stimulate the retina by shining light through the sclera. In this way the opacities are circumvented but light must now pass through the choroid and pigment epithelium to reach the receptors. One might expect a considerable variation of light transmission depending on the complexion and pigmentation of the patient. In spite of this, useful results have been obtained using this kind of technique. ${ }^{21}$

Electrodiagnostic tests in the presence of a cataract

It has already been shown that if a flash ERG alone is measured preoperatively, then a normal result does not exclude the possibility of postoperative blindness from glaucoma or a central scotoma from macula degeneration. It is equally possible to regain a good postoperative visual acuity when the routinely recorded ERG has been flat preoperatively. This could occur if a small area of functioning retina remained at the fovea. Babel et al have claimed that a more reliable result from the prognostic point of view can be obtained if the ERG to a red flash is used in combination with the flicker ERG. They claim that this gives a better chance of detecting abnormal macula function. ${ }^{22}$ Recently it has been claimed that a combination of flicker ERG and flicker VEP can be used to exclude optic nerve damage and macular disease in preoperative cataract patients and patients with vitreous haemorrhage. The same authors claim that the flicker VEP behaves quite independently from the flicker ERG and even that the flicker
ERG may be absent and yet the flicker VEP be normal. Thus they have recarded a normal flicker VEP when the ERG was markedly impaired by retinal detachment. ${ }^{23}$ The value of the flash VEP as an adjunct to the ERG is becoming more widely recognised ${ }^{24}$ Thompson and Harding in their study of 21 eyes were able to show postoperative follow up in 20 of them. The study was limited to the VEP and they showed that the VEP could predict well except in cases of amblyopia. ${ }^{20}$ The flash as opposed to the pattern VEP is not affected by amblyopia. It is not possible at present to detect amblyopia of disuse through opaque media by electrodiagnostic means. An attempt has been made, with partial success, to record pattern VEPs behind a cataract using laser interference fringes. A VEP could be recorded if the cataract was not too dense, but the method involved technical difficulties especially with elderly patients. ${ }^{25}$ Some of the questions that we might ask prior to surgery on a patient with a dense cataract usually concern the possibility of senile macula degeneration, amblyopia of disuse, diabetic retinopathy or glaucomatous damage existing behind the cataract. A firm knowledge of their existence would not necessarily contraindicate surgery, but it would help to provide the patient as well as the surgeon with a more accurate prognosis. Diabetic retinopathy of any severity can usually be predicted by the absence of wavelets in the flash ERG. ${ }^{26}$ These oscillations are seen on the ' $b$ ' wave of the normal ERG with suitable stimulus conditions. They are known to be independent of the ' $b$ ' wave and are selectively reduced in size by impairment of the retinal circulation. ${ }^{27}$ A significant correlation has been shown between the amplitude of the wavelets and other visual function tests including colour vision, fluorescein leakage and visual acuity. ${ }^{28}$ Although loss or reduction of the wavelets is an early change, as the disease advances the ' $a$ ' and ' $b$ ' waves also become reduced. In very severe proliferative disease the ERG may be abolished. Figure 3 shows the ERG of a patient who was blind from severe diabetic retinopathy and cataract. A flash VEP was not performed but cataract surgery 
was carried out in spite of the poor ERG. This proved to have been the right decision because a vision of $6 / 9$ was achieved postoperatively. A small island of functioning retina remained at the macula.

D.P. 19.4 .83

Right

Eye

Left
Eye

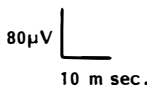

Fig. 3. Absent response (ERG) in a patient who subsequently underwent cataract surgery with a good visual result.

E.B. 23.11 .82
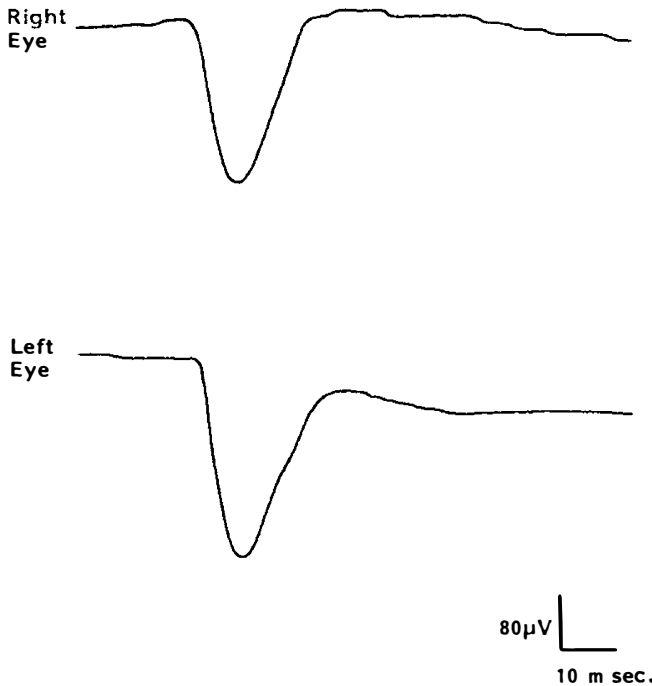

Fig. 4. ERGs from a patient with bilateral cataracts, chronic glaucoma, and central retinal vein occlusions.
The case illustrates the importance of doing a flash VEP in combination with the ERG in these circumstances. The wavelets on the ' $b$ ' wave may be affected by other forms of vascular disease and they also diminish with age. A unilateral loss of wavelets in the presence of a vitreous haemorrhage suggests a central retinal vein occlusion. Figure 4 shows bilateral loss of wavelets in a patient with chronic glaucoma and bilateral central retinal vein occlusions. The fundi were partially obscured by cataracts and the vision was 'Counts fingers' in the right eye and $6 / 60$ in the left eye. Senile macular degeneration is not easy to detect behind a cataract if the damage is limited to a small central area. The flash VEP should, at least, in theory be useful in this respect and although this also appears to be the case in clinical practice a good series of cases giving pre- and post-operative results has yet to be published. Loss of sight from glaucomatous damage of the optic nerve can be inferred from the light projection test preoperatively but, of course, the flash ERG may be completely normal. If the ERG is backed up with the flash VEP then the damage should become evident. In the absence of opaque media the amplitude of the first positive peak of the VEP can be a sensitive indicator of optic nerve damage in glaucoma. It has been suggested that the VEP shows changes before loss of visual field. ${ }^{29}$ Presumably, similar changes might be detected through cataracts if the brightness of the flash were adequate.

Electrodiagnostic tests in the presence of a vitreous haemorrhage

Vitreous haemorrhage is a good indication for a flash ERG and VEP if applied to the right patients. A common example is the diabetic patient. Scherfig et al examined 116 diabetic eyes prior to vitreous surgery. Using their technique, if the first positive peak of the VEP normally seen at $70 \mathrm{msec}$ was delayed beyond $100 \mathrm{msec}$ then the chances of obtaining an improvement in vision were small. Only two of the fifty-one patients who showed a visual improvement after surgery had a latency longer than $100 \mathrm{msec}$ (see Fig. 5). In four cases the flash ERG was extinguished when the flash VEP was present 
suggesting severe retinal damage or detachment but reasonable macula function. ${ }^{30}$ Other authors have also claimed that the VEP in particular can be a good predictor of visual outcome in vitreous haemorrhage patients. ${ }^{31,32}$ In diabetic patients it is important to be aware of the effect of photocoagulation on the electrical responses. The flash ERG can be considerably reduced in amplitude and the relationship of amplitude of ' $a$ ' wave and ' $b$ ' wave can also be disturbed. ${ }^{33,34}$

A further point of confusion arises from the fact that the ERG may be diminished after any severe injury to the eye in relation to the extent of commotio retinae. The effect is well recognised and may last for a week or more. ${ }^{11}$

After severe eye injuries, a combination of flash ERG and flash VEP can give useful results. In one series one hundred patients were tested prior to vitrectomy and the ERG was shown to be the most useful indicator of postoperative visual acuity. The VEP gave similar but less reliable results. ${ }^{35}$ Crews et al examined 64 eyes of 60 patients with severe perforating and blunt injuries. They conclude
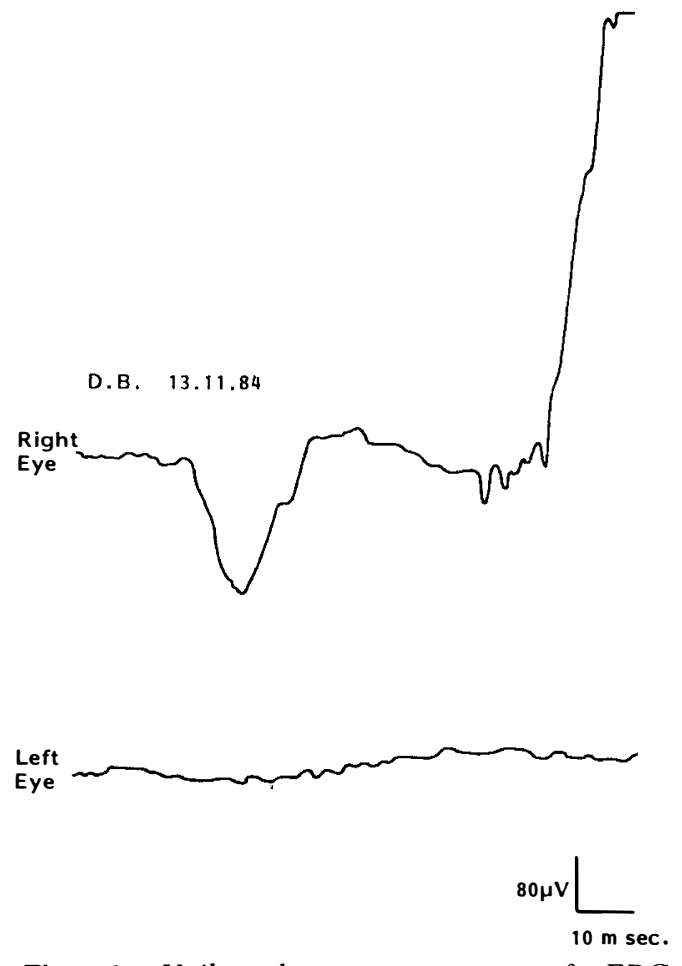

Fig. 6. Unilateral poor response of $E R G$ suggesting a retinal detachment.

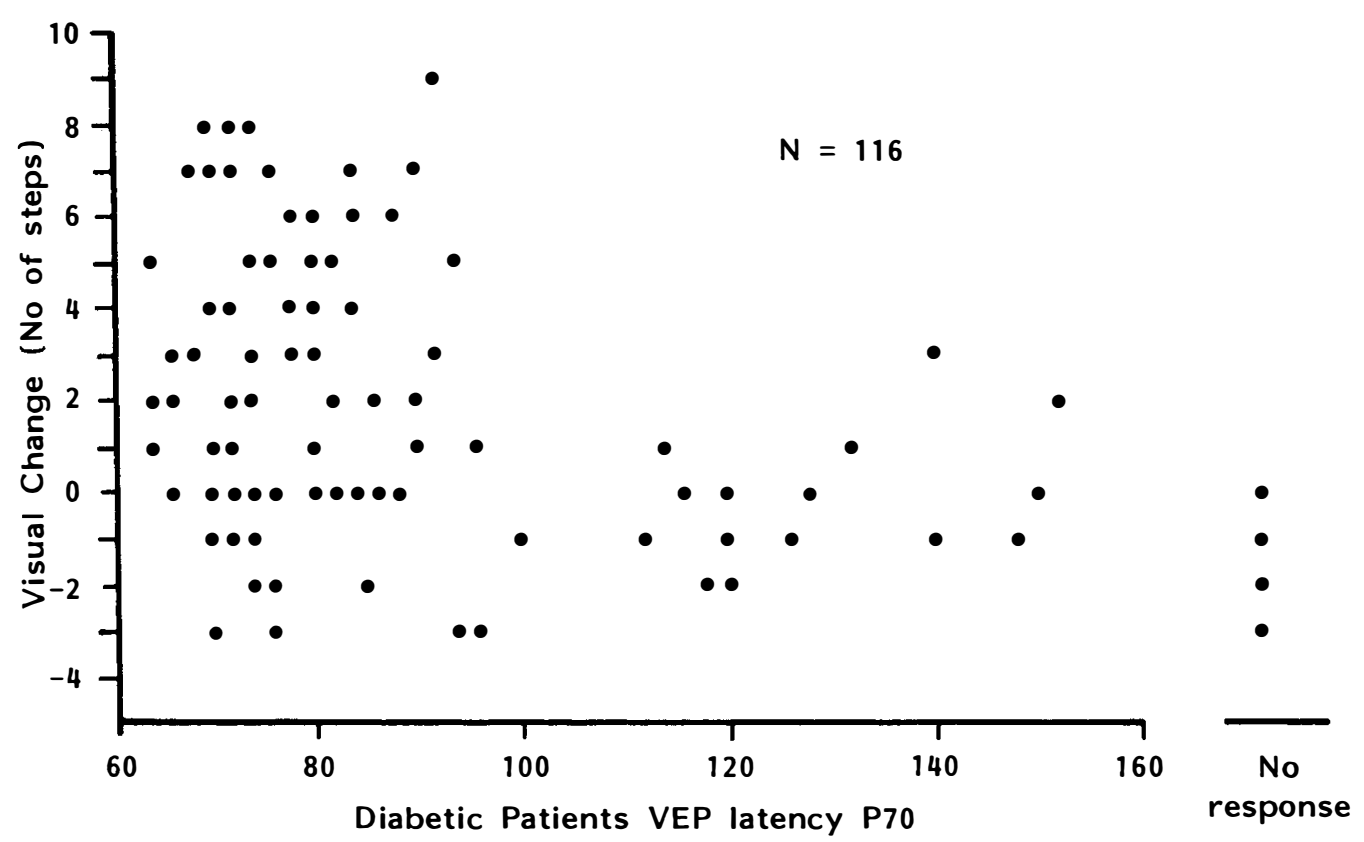

Fig. 5. Relationship of visual improvement after surgery to flash VEP amplitude (Scherfig et al 1984). 
that the combination of the ERG and VEP offers a diagnostic enhancement over other tests. In their series, if the 'b' wave of the ERG was not reduced more than $50 \%$ in amplitude, and if the VEP amplitude was also less than $50 \%$ reduced and without any delay, then a good visual result was invariably predicted. ${ }^{36}$

Electrodiagnostics can be usefully applied to patients with vitreous haemorrhage of unknown cause. Figure 6 shows the response from a patient with a presumed retinal detachment. He had suffered a perforating injury five months previously. A cataract had developed and there was a dense vitreous haemorrhage. The electrodiagnostic results indicate a likely total retinal detachment. It is well recognised that the ERG is diminished immediately by a detachment and the reduction in amplitude is related to the extent of the detachment. ${ }^{37}$

A considerable interest has, in recent years, centred on the effect of silicone oil replacement of the vitreous on the ERG. A reduction in the amplitude of the ERG has been reported and evidence is now appearing which suggests that this reduction is due to an insulating effect of the silicone oil rather than to any toxic effect on the retina. The ERG appears to recover as soon as the silicone oil is removed. ${ }^{38,39,40,41}$ No similar reduction in the response has been recorded after the use of intraocular lens implants.

Electrodiagnosis in the presence of corneal opacities

The prognosis following corneal grafting has improved greatly in recent years due to advances in donor tissue evaluation and surgical instrumentation as well as to the introduction of drugs modifying the immune graft reaction. In spite of this the preoperative evaluation of visual potential is still a problem. There has been some recent interest in the use of electrodiagnostics for this purpose and the results are similar to

WITH $\rightarrow$ AFTER SILICONE OIL

EOC

Basic Value

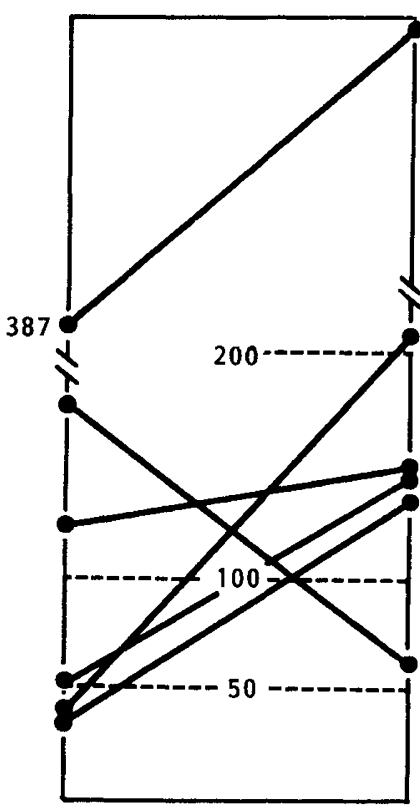

ERG

Scotopic

757

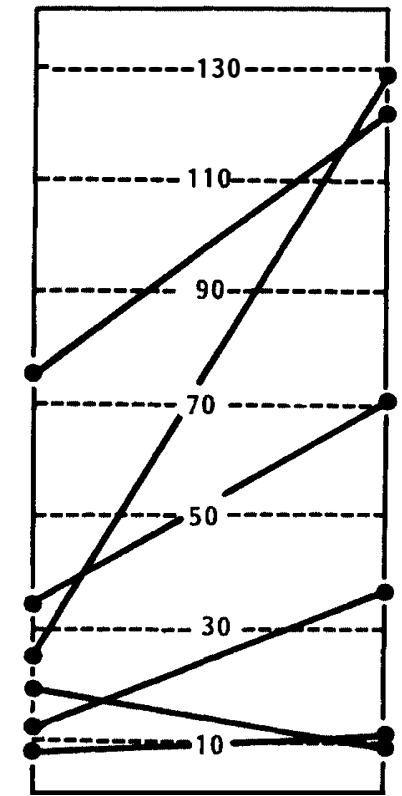

ERG

Photopic

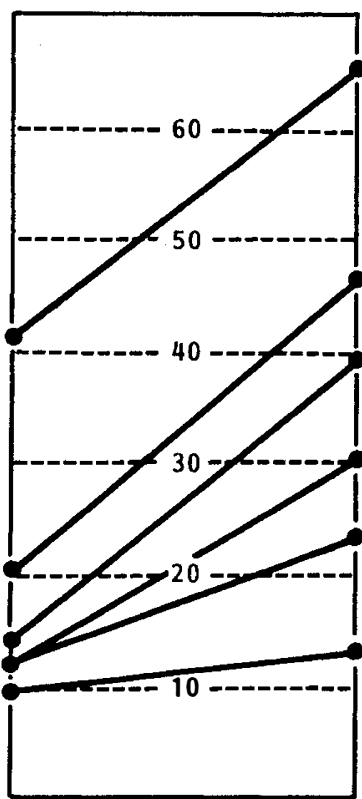

Fig. 7. Increase in amplitude of the ERG and EOG which may follow removal of silicone oil. (Momirov et al 1983.) 
those obtained in other types of medial opacity.

In a series of 32 patients, Wendel and coworkers compared pre-operative flash VEPs and ERGs with postoperative clinical findings and claimed that they were able to predict the visual outcome in $92 \%$ of cases. ${ }^{42}$ Inaccurate prediction occurred in a patient who was subsequently found to have cystoid macular oedema and in another with a history of amblyopia. All those patients predicted to do poorly did so. These authors stress the frequent finding of an increase in amplitude of both ' $a$ ' and ' $b$ ' waves in the presence of corneal opacities. This was presumably due to the "Ganzfeld effect" as described above. One of these patients had good electrodiagnostic results in the presence of an afferent pupillary defect. This patient recovered some useful vision in spite of the preoperative clinical signs. A further important point born out by this series is the value of comparing the two eyes when one is normal clinically. This greatly enhances the predictive value of eletrodiagnostic testing. ${ }^{43}$

Electrodiagnostic tests of visual function are of undoubted value when applied to eyes with opaque media and suspected retinal disease. The combined investigation of the flash ERG and the VEP has been shown to be of real practical value. This combination of tests makes it possible to distinguish central and peripheral retinal disease. One might wonder why these tests are not widely usẽd in patients with medial opacities. One possible reason is the lack of standardisation which has existed in the past. This has meant that the results from different clinics are not comparable. Electroretinographic techniques are in the process of being standardised and during the course of the ensuing year this bar to progress should be overcome.

\footnotetext{
References

${ }^{1}$ Scheerer R: die Entoptische Sichtbarkeit der Blutbewegung im Auge und ihre klinische Bedeutung. Klin Monatsb Augenheilkd 1924; 73: 67-107.

2 Sinclair SH, Loebl M, Riva CE: Blue field entoptic phenomenon in cataract patients. Arch Ophthalmol 1979; 97: 1092-5.

${ }^{3}$ Gstalder RJ and Green DG: Laser
}

interferometry in corneal opacification. Arch Ophthalmol 1972; 87: 269-74.

${ }^{4}$ Faulkner W: Laser interferometric prediction of postoperative acuity in patients with cataracts. Am J Ophthalmol 1983; 96: 626-36.

${ }^{5}$ Armington JC: 'The Electroretinogram' 1974. p.402-405. Academic Press. New York, San Francisco, London.

${ }^{6}$ Feinsod M, Rowe H, Auerbach E: Changes in the Electroretinogram in patients with optic nerve lesions. Doc Ophthalmol 1971, 29: 169200.

${ }^{7}$ Galloway NR: 'Ophthalmic Electrodiagnosis' 1981. p.86-147. Lloyd Luke Ltd. London.

${ }^{8}$ Halliday AM: Evoked potentials in clinical testing 1982. p99 Churchill Livingstone. Edinburgh, London, Melbourne, New York.

${ }^{9}$ Adrian ED: Rod and cone components in the electric response of the eye. J Physiol 1946, 105: 24-37.

${ }^{10}$ Karpe G: Flicker electroretinography. Ophthalmologica Belgica XVIII Concilium 1958: 607-14.

${ }^{11}$ Knighton RW and Blankenship GW: Int Ophthalmol Clinics 1980; 20:1-19. Little, Brown \& Co, Boston.

${ }^{12}$ Burian HM and Burns CA: A note on senile cataracts and the electroretinogram. Doc Ophthalmol 1966; 20: 141.

${ }^{13}$ Mikawa $T$ and Tamura O: Relation between the electroretinogram and post operation vision in cataract. Jpn J Clin Ophthalmol (Rinsho Ganka) 1970; 24: 43-6.

${ }^{14}$ Hess RF and Garner LF: The effect of corneal oedema on visual function. Invest Ophthalmol 1977; 16: 5-13.

${ }^{15}$ Hess RF and Woo G: Vision through cataracts. Invest Ophthalmol 1978; 17: 428-35.

${ }^{16}$ LeClaire J, Nadler P, Weiss S, Miller D: A new glare tester for clinical testing. Results comparing normal subjects and variously corrected aphakic patients. Arch Ophthalmol 1982; 100: 153-8.

${ }^{17}$ Copenhaver RM and Perry NW: Factors affecting visualiy evoked cortical potentials such as impaired vision of varying aetiology. Invest Ophthalmol 1964; 3: 665-75.

${ }^{18}$ Skalka $\mathrm{H}$ and Holman J: Effect of pupillary dilatation in flash VER testing. Doc Ophthalmol 1986; 63: 321-4.

${ }^{19}$ Holder GE and Huber MJE: The effect of miosis on pattern and flash ERG and pattern Visual Evoked Potential. Doc Ophthal Proc Series 1984; 40: 109-16.

${ }^{20}$ Thompson CRS and Harding GFA: The visual evoked potential in patients with cataracts. Doc Ophthalmol Proc Series 1978; 15: 193. 
${ }^{21}$ Rubin ML and Dawson WW: The trans-scleral VER: Prediction of postoperative acuity. Invest Ophthalmol Visual Sci 1978; 17: 71.

${ }^{22}$ Babel J, Stangos N, Korol S, Spiritus M: Ocular Electrophysiology: A clinical and experimental study of electroretinogram, electro-oculogram and visually evoked response. Stuttgart. George Thieme Publishers. 1977; 1-172.

${ }^{23}$ Foerster $\mathrm{MH}$ and $\mathrm{Li}$ XX: Evaluation of the central retina and optic nerve function in media opacities. Doc Ophthalmol, 1986; 63: $101-6$.

${ }^{24}$ Vrijland HR and van Lith GHM: The value of preoperative electro-ophthalmological examination before cataract extraction. Doc Ophthalmol, 1983; 55: 153-6.

${ }^{25}$ Arden GB and Sheory UB: The assessment of visual function in patients with opacities. A new evoked potential method using a laser interferometer. In Desmedt JE (Ed) Visual Evoked Potential in Man: New Developments. Oxford: Clarendon 1977; p381-94.

${ }^{26}$ Simonsen SE: The value of the oscillatory potential in selecting juvenile patients at risk of developing proliferative retinopathy. Acta Ophthalmol 1980; 58: 865-78.

27 Wachmeister L: Basic research and clinical aspects of the oscillatory potential of the electroretinogram. Doc Ophthalmologica, 1987; 66: 187-94.

${ }^{28}$ Bresnick GH and Palta M: Oscillatory potential amplitudes. Relation to severity of diabetic retinopathy. Arch Ophthalmol, 1987; 105: 929-33.

${ }^{29}$ Good PA, Marsters JB, Mortimer MJ: Flash stimulation evoked potentials in diagnosis of chronic glaucoma. Lancet, ii: 1987; 1259-60.

${ }^{30}$ Scherfig E, Edmund J, Tinning S, Trojaborg W: Flash visual evoked potential as a prognostic factor for vitreous operations in diabetic eyes. Ophthalmology, 1984; 91: 1475-9.

${ }^{31}$ Tolentino F, Schepens CL, Freeman HM: Vitreoretinal disorders; Diagnosis and management. Philadelphia: W. B. Saunders, 1976; 539-9.

${ }^{32}$ Fuller DG and Hutton WL: Presurgical evaluation of eyes with opaque media New York. Grune \& Sttratton, 1982; 48-60.

${ }^{33}$ Perlman I, Gdal-on M, Miller B, Zonis S: Retinal function of the diabetic retina after argon laser photocoagulation assessed electroretinographically, $\mathrm{Br} J$ Ophthalmol, 1985; 69: 240-6.

${ }^{34}$ François J, De Rouck A, Cambie E, Castanheira-Dinis A: Electrophysiological studies before and after argon laser photocoagulation in diabetic retinopathy. Ophthalmologica, 1978: 176: 133-44.

${ }^{35}$ Bach $\mathrm{M}$ and Rover J: Der prognostische Wert von ERG, Ultraschall und VEP bei Glaskorperblutungen. Fortsch Ophthalmol 1984; 81: 274-6.

${ }^{36}$ Crews SJ, Thompson CRS, Harding GFA: The ERG and VEP in patients with severe eye injury. Doc Ophthalmol Proc Series, 1978; 15: 203-9.

${ }^{37}$ Rendahl I: The clinical electroretinogram in detachment of the retina. Acta Ophthalmol, 1961; 64: 1-83.

${ }^{38}$ Frumar KD, Gregor ZJ, Carter RM, Arden GB: Electroretinographic changes after vitrectomy and intraocular tamponade. Retina, 1985; 5: 16-21.

${ }^{39}$ Momirov D, Van Lith GHM, Zivojnovic R: Electroretinogram and electro-oculogram of eyes with intravitreously injected silicone oil. Ophthalmologica. 1983; 186: 183-8.

${ }^{40}$ Meredith TA, Lindsey DT, Edelhauser HF, Goldman AI: Electroretinographic studies following vitrectomy and intraocular silicone oil injection. Br J Ophthalmol, 1985; 69: 25460.

${ }^{41}$ Foerster MH, Esser J, Laqua H: Silicone oil and its influence on electrophysiological findings. Am J Ophthalmol, 1985; 99: 201-6.

${ }^{42}$ Wendel RT, Mannis MJ, Keltner JL. Role of electrophysiologic testing in the preoperative evaluation of corneal transplant patients. Ann Ophthalmol, 1984; 16: 788-93.

${ }^{43}$ Binder PS, Avazuddin M, Hintze R: Visual prognosis for corneal transplantation based on preoperative visual evoked potential and electroretinogram. Ophthalmology, 1982; 89: 661-2. 